\title{
Evaluation of single-view contrast-enhanced mammography as novel reading strategy: a non-inferiority feasibility study
}

\author{
M. B. I. Lobbes ${ }^{1,2}$ D • J. Hecker ${ }^{1}$ ・ I. P. L. Houben ${ }^{1} \cdot$ R. Pluymakers ${ }^{1} \cdot$ C. Jeukens $^{1} \cdot$ U. C. Laji ${ }^{1} \cdot$ S. Gommers $^{1} \cdot$ \\ J. E. Wildberger ${ }^{1}$. P. J. Nelemans ${ }^{3}$
}

Received: 10 January 2019 /Revised: 13 March 2019 / Accepted: 2 April 2019/Published online: 9 May 2019

(C) The Author(s) 2019

\begin{abstract}
Background Guidelines recommend screening of high-risk women using breast magnetic resonance imaging (MRI). Contrastenhanced mammography (CEM) has matured, providing excellent diagnostic accuracy. To lower total radiation dose, evaluation of single-view (1 V) CEM exams might be considered instead of double-view (2 V) readings as an alternative reading strategy in women who cannot undergo MRI.

Methods This retrospective non-inferiority feasibility study evaluates whether the use of $1 \mathrm{~V}$ results in an acceptable sensitivity for detecting breast cancer (non-inferiority margin, - 10\%). CEM images from May 2013 to December 2017 were included. $1 \mathrm{~V}$ readings were performed by consensus opinion of three radiologists, followed by $2 \mathrm{~V}$ readings being performed after 6 weeks. Cases were considered "malignant" if the final BI-RADS score was $\geq 4$, enabling calculation of sensitivity, specificity, and area under the receiver operating characteristic curve (AUC). Histopathological results or follow-up served as a gold standard.

Results A total of 368 cases were evaluated. Mean follow-up for benign or negative cases was 20.9 months. Sensitivity decreased by $9.6 \%$ from 92.9 to $83.3 \%$ when only $1 \mathrm{~V}$ was used for evaluation $(p<0.001)$. The lower limit of the $90 \%$ confidence interval around the difference in sensitivity between $1 \mathrm{~V}$ and $2 \mathrm{~V}$ readings was $-15 \%$ and lies below the predefined non-inferiority margin of $-10 \%$. Hence, non-inferiority of $1 \mathrm{~V}$ to $2 \mathrm{~V}$ reading cannot be concluded. AUC for $1 \mathrm{~V}$ was significantly lower, 0.861 versus 0.899 for $2 \mathrm{~V}(p=0.0174)$.

Conclusion Non-inferiority of $1 \mathrm{~V}$ evaluations as an alternative reading strategy to standard $2 \mathrm{~V}$ evaluations could not be concluded. $1 \mathrm{~V}$ evaluations had lower diagnostic performance compared with $2 \mathrm{~V}$ evaluations.

Key Points

- To lower radiation exposure used in contrast-enhanced mammography, we studied a hypothetical alternative strategy: singleview readings $(1 \mathrm{~V})$ versus (standard) double-view readings $(2 \mathrm{~V})$.

- Based on our predefined margin of - 10\%, non-inferiority of $1 \mathrm{~V}$ could not be concluded.

- 1 Vevaluation is not recommended as an alternative reading strategy to lower CEM-related radiation exposure.
\end{abstract}

Keywords Breast cancer · Screening · Mammography

M. B. I. Lobbes

marc.lobbes@mumc.nl

1 Department of Radiology and Nuclear Medicine, Maastricht University Medical Center, P.O. Box 5800, 6202

AZ Maastricht, The Netherlands
2 GROW School for Oncology and Developmental Biology, Maastricht University, Maastricht, The Netherlands

3 Department of Epidemiology, Maastricht University, Maastricht, The Netherlands 


$\begin{array}{ll}\text { Abbreviations } \\ \text { 1 V } & \text { Single-view contrast-enhanced mammography } \\ \text { 2 V } & \text { Double-view contrast-enhanced mammography } \\ \text { AUC } & \text { Area under ROC curve } \\ \text { CEDM } & \text { Contrast-enhanced dual-energy mammography } \\ \text { CEM } & \text { Contrast-enhanced mammography } \\ \text { CESM } & \text { Contrast-enhanced spectral mammography } \\ \text { CI } & \text { Confidence interval } \\ \text { DCIS } & \text { Ductal carcinoma in situ } \\ \text { FFDM } & \text { Full-field digital mammography } \\ \text { FN } & \text { False negative } \\ \text { FP } & \text { False positive } \\ \text { ILC } & \text { Invasive lobular carcinoma } \\ \text { MRI } & \text { Magnetic resonance imaging } \\ \text { NPV } & \text { Negative predictive value } \\ \text { NST } & \text { No special type } \\ \text { PPV } & \text { Positive predictive value } \\ \text { ROC } & \text { Receiver operating characteristic } \\ \text { TN } & \text { True negative } \\ \text { TP } & \text { True positive }\end{array}$

\section{Introduction}

Breast cancer is a leading cause of cancer-related deaths in women worldwide every year. Some women have genetic mutations, making them more susceptible to develop breast cancer in their life. These include, for example, BRCA-1, BRCA-2, TP53, PALB2, CDH1, STK11, and PTEN gene mutations. Other reasons for having a $>20 \%$ lifetime risk of developing breast cancer include prior chest (mantle) radiation and specific syndromes, such as Li Fraumeni or Cowden syndrome. Based on studies that showed an improved cancer detection rate in these women when breast MRI is used as adjunct screening modality [1-3], current international guidelines recommend annual screening of these women with breast MRI [4-6].

The use of breast MRI as a screening tool has some limitations. It is a relatively expensive modality and a widespread use as a screening tool is challenging due to the limited availability of sufficient scan slots. Breast MRI has an excellent sensitivity, but its specificity is moderate (resulting in falsepositive findings requiring additional follow-up exams or biopsies) [7]. In addition, studies have shown that gadolinium (Gd) of contrast agents accumulates in the body [8]. Although no negative long-term side-effects have been reported, this phenomenon might result in a discouragement of using Gdbased contrast agents for (repeated) screening purposes. Finally, a number of women will not be able to undergo breast MRI because of claustrophobia, previous adverse reactions to the contrast agent used, or the presence of metal objects within their bodies. Therefore, an alternative imaging modality might be appealing for these groups of women, using for example contrast-enhanced mammography (CEM, synonyms: CESM, contrast-enhanced spectral mammography or CEDM, contrast-enhanced dual-energy mammography).

The underlying principle of CEM is comparable to that of breast MRI: growing tumors need to sprout newly formed blood vessels to adhere to their increasing demand for nutrients in a process called angiogenesis [9]. These newly formed vessels are rapidly formed and "leaky" to contrast agents like the ones used in CEM or breast MRI [10]. These contrast agents can extravasate into the tumor interstitium, causing enhancement on CEM or MRI exams. Multiple studies have evaluated the diagnostic performance of CEM compared with breast MRI, showing that sensitivity is at least equal to breast MRI [11-13]. CEM is increasingly considered as a potential screening modality, especially in women with high or intermediate breast cancer risk or dense breasts $[14,15]$. However, disadvantages of CEM over breast MRI, especially when it would be considered in screening of patients, are not only the use of iodinated contrast agents but also its increased radiation dose (for example, when compared with full-field digital mammography or FFDM) [16] and the lack of CEM-guided biopsy capabilities.

To compensate for the increased radiation dose in screening patients at high risk for developing breast cancer using CEM, we propose an alternative strategy: single-view CEM. In this retrospective study, we evaluated the diagnostic performance of single-view CEM ( $1 \mathrm{~V})$ versus standard double-view CEM $(2 \mathrm{~V})$ to find out if this approach has the potential to serve as an alternative strategy. This retrospective study was designed as a non-inferiority study to evaluate whether use of $1 \mathrm{~V}$ results in an acceptable sensitivity for detecting breast cancer in our study population, while maintaining similar specificity when compared with $2 \mathrm{~V}$. However, we also consider this to be a feasibility study, as our primary analyses were not conducted on the assumed target population of women at high risk of developing breast cancer, but on our institute's available CEM database.

\section{Materials and methods}

For this study, we retrospectively analyzed all CEM exams performed at our institute between May 2013 and December 2017. Indications for CEM included recalls after a positive screening mammography, suspicious findings during physical examination or detected on imaging performed elsewhere, unknown primary tumors, and inconclusive findings at FFDM or alternative to breast MRI. All images were anonymized with an allocated study code. Due to the study design used, the necessity to acquire informed consent was waived by our ethical committee (decision number METC 15-4-008). 


\section{Image protocol and gold standard}

All CEM exams were performed on a single CEM unit (Senographe* Essential with Senobright* upgrade, GE Healthcare) using a non-ionic, monomeric, low-osmolar contrast agent at a dose of $1.5 \mathrm{ml} / \mathrm{kg}$ of body weight (iopromide, Ultravist 300, Bayer Healthcare). Iodinated contrast was administered intravenously with a flow rate of $3 \mathrm{ml} / \mathrm{s} 2 \mathrm{~min}$ prior to image acquisition. The breasts were imaged in (at least) mediolateral oblique and craniocaudal views.

For all solid lesions and (micro)calcifications, histopathological results served as the gold standard. In cases of negative results or suspected cysts, a minimum follow-up of 12 months was used to exclude any false-negative findings.

\section{Image analysis}

The CEM images were evaluated using a double-reading strategy, which is the applied strategy for our nationwide screening program. In short, two certified screening radiologists (with 9 and 7 years of screening expertise and both having 5 years of CEM experience) provided a BI-RADS classification for the $1 \mathrm{~V}$ images first (i.e., mediolateral oblique view for both breasts, as this view covers the largest part of the breast). For this study, we considered a BI-RADS classification of $\geq 4$ to be "suspicious for breast cancer," which in a screening setting would require a recall. BI-RADS classifications $\leq 3$ were considered "not suspicious for breast cancer" and would not have been recalled in a screening setting. For the primary analysis, a consensus opinion was used and in case of discrepancies between both readers, a third screening-certified reader (6 years of screening expertise and 5 years of CEM experience) was consulted for the final decision. To minimize recall bias, all cases were re-evaluated in a similar fashion after 6 weeks, but this time, the complete CEM exam was available ( $2 \mathrm{~V})$. The images were evaluated in a different, randomized order in these two sessions. During the evaluations, all radiologists were blinded to the primary CEM indication, their score in the other reading session and final diagnosis.

\section{Statistical analysis}

The study was designed as a non-inferiority study to evaluate whether the use of single-view CEM exams does not result in an unacceptable lower sensitivity for detecting breast cancer in our study population, while maintaining similar specificity when compared with double-view CEM exams. The prespecified non-inferiority margin was determined at $10 \%$. This margin was chosen because a sensitivity decrease by more than $10 \%$ was considered unacceptable.

Assuming a sensitivity in the reference group (i.e., $2 \mathrm{~V}$ group) for the detection of malignant cases of $90 \%, 112$ pairs with malignant cases are required to be $80 \%$ sure that a one- sided $95 \%$ confidence interval (CI, equivalent to a two-sided $90 \% \mathrm{CI}$ ) will exclude a difference in favor of the $2 \mathrm{~V}$ group of more than $10 \%$. Prior to this study, we estimated the prevalence of malignant cases to be $30 \%$ [17], resulting in a required total sample size of 373 cases (source: OpenEpi, www.openepi.com).

As explained before, BI-RADS 1-3 were considered benign and BI-RADS 4-5 malignant. Using these cutoff values, sensitivity, specificity, positive predictive value (PPV), and negative predictive value (NPV) were calculated. In addition, the area under the ROC curve (AUC) was calculated for the different reading sessions.

The absolute differences in sensitivity and specificity and one-sided 95\% CI (equivalent to two-sided 90\% CI) of the difference were calculated using Tango's score CI for a difference of paired proportions [18]. The corresponding one-sided $p$ values were derived using McNemar's test for paired proportions. The paired areas under the curve (AUC) of the receiver operating characteristic (ROC) curves for $1 \mathrm{~V}$ and $2 \mathrm{~V}$ view exams were compared using an algorithm developed by DeLong et al [19].

STATA (version 13.1, StataCorp LLC) and R (version 2.15.1, The R Foundation for Statistical Computing) were used for the statistical analyses. One-sided $p$ values of 5\% were considered to indicate statistical significance.

\section{Results}

The mean age of the women included in our study population was 59.7 years (range $50-77$ years). In the study period, 368 patients instead of the required 373 patients were included, but the prevalence of malignancies turned out to be higher than expected $(34.2 \%)$. Consequently, the number of malignant cases was 126, which is higher than the planned sample size of 112 cases for the evaluation of the sensitivity difference. Of the 126 malignant diagnoses, 48 consisted of invasive cancer of no special type (NST, or invasive ductal carcinoma; 13\%), followed by 42 cases of ductal carcinoma in situ (DCIS, $11.4 \%$ ) and 30 cases of invasive lobular carcinoma (ILC, $8.1 \%$ ). The remaining 6 cases were invasive breast cancers not otherwise specified (1.7\%). Of the benign diagnoses, most were cysts $(n=90)$, followed by fibroadenoma $(n=19)$ and intramammary lymph nodes $(n=10)$, with the remaining benign diagnosis being not otherwise specified $(n=35)$. A total of 88 cases were negative. The mean follow-up period for benign or negative cases was 20.9 months, range 12.755.3 months.

The results for the $1 \mathrm{~V}$ and the $2 \mathrm{~V}$ evaluation are presented in Table 1. For the $1 \mathrm{~V}$ readings, there were 48 discrepancies between the first two readers, while the number of discrepancies for the $2 \mathrm{~V}$ readings was 64 . The combined assessment of the radiologists based on $1 \mathrm{~V}$ CEM images resulted in 105 
Table 1 Comparison of sensitivity and specificity between double-view ( $2 \mathrm{~V})$ and single-view (1 V) contrast-enhanced mammography exams. Results are presented for the combination of all three readers $(\mathrm{R} 1+\mathrm{R} 2+\mathrm{R} 3)$ and for the first $(\mathrm{R} 1)$ and second $(\mathrm{R} 2)$ reader independently

\begin{tabular}{lllll}
\hline & Double $(2 \mathrm{~V})$ view $\%(n)$ & Single $(1 \mathrm{~V})$ view $\%(n)$ & Difference \% 1 V minus 2 V (90\% CI) & $p$ value (one-sided) \\
\hline Sensitivity & & & & $<0.001$ \\
R1 + R2 + R3 & $92.9(117 / 126)$ & $83.3(105 / 126)$ & $-9.6(-15$ to -5.3$)$ & $<0.001$ \\
R1 & $92.1(116 / 126)$ & $76.2(96 / 126)$ & $-15.9(-22$ to -10$)$ & 0.0012 \\
R2 & $92.9(117 / 126)$ & $82.5(104 / 126)$ & $-10.4(-16$ to -5$)$ & \\
Specificity & & & $+5.0(1.2$ to 8.9$)$ & 0.0251 \\
R1 + R2 + R3 & $79.3(192 / 242)$ & $84.3(204 / 242)$ & $+18.6(14$ to 23$)$ & $<.001$ \\
R1 & $65.7(159 / 242)$ & $84.3(204 / 242)$ & $+4.2(-0.1$ to 8.5$)$ & 0.07165 \\
R2 & $72.7(176 / 242)$ & $76.9(186 / 242)$ & \\
\hline
\end{tabular}

CI, confidence interval

true-positive cases (TP), 38 false-positive cases (FP), 204 truenegative cases (TN), and 21 false-negative cases (FN). For the 2 Vevaluation, these numbers were the following: 117 TPs, 50 FPs, 192 TNs, and 9 FNs, respectively. An overview of the histopathological results of the FN cases is presented in Table 2. An example of an invasive lobular carcinoma that was overlooked on the $1 \mathrm{~V}$ (MLO) exam and detected on $2 \mathrm{~V}$ readings is presented in Fig. 1.

Our results show that sensitivity decreases when only $1 \mathrm{~V}$ is used for the evaluation of the CEM images. This decrease is statistically significant $(p<0.001)$. The lower limit of the $90 \%$ $\mathrm{CI}$ of the difference in sensitivity between $1 \mathrm{~V}$ and $2 \mathrm{~V}$ readings is $-15 \%$ and lies below $-10 \%$, but the entire $90 \% \mathrm{CI}$ (ranging from -15 to $-5.3 \%$ ) does not exclude the non-inferiority margin. More specifically, non-inferiority could be observed for the first reader, but could not be concluded for the second or the combined readings. It can also be observed that there is a tradeoff between sensitivity and specificity: the significant decrease of sensitivity is accompanied by a significant increase in specificity. These trends are observed for the evaluations by R1 and R2 as well. To evaluate whether overall diagnostic performance decreases when using the $1 \mathrm{~V}$ strategy instead of the standard $2 \mathrm{~V}$ evaluation, AUCs were compared. The ROC curves for the consensus results $(\mathrm{R} 1+\mathrm{R} 2+\mathrm{R} 3$ based on $1 \mathrm{~V}$ and $2 \mathrm{~V}$ readings) are presented in Fig. 2. The AUC for $1 \mathrm{~V}$ was 0.861 versus 0.899 for $2 \mathrm{~V}$ (one-sided $p=0.0174$ ), indicating a significantly worse overall diagnostic performance for the $1 \mathrm{~V}$ readings when compared with $2 \mathrm{~V}$.

These results, however, were based on the analyses performed on our institute's CEM database, which is not the assumed target population of women at high risk for developing breast cancer (see also the "Study limitations" section).

\section{Discussion}

In this study, the sensitivity of $1 \mathrm{~V}$ evaluations was significantly lower compared with (standard) $2 \mathrm{~V}$ evaluations. The lower
CI limit of the difference in sensitivity between $1 \mathrm{~V}$ and $2 \mathrm{~V}$ lies below the predefined non-inferiority margin of $-10 \%$ and, consequently, the results did not allow for the conclusion that $1 \mathrm{~V}$ evaluation is non-inferior to $2 \mathrm{~V}$ evaluation of CEM exams. We observed a trade-off between sensitivity and specificity: the significant decrease of sensitivity was accompanied by a significant increase in specificity. These trends were also observed for the evaluations by R1 and R2. Using $1 \mathrm{~V}$ readings instead of $2 \mathrm{~V}$ readings leads to a substantial and significant decrease of sensitivity and overall diagnostic performance. The majority of FN diagnoses were caused by DCIS and ILC (Table 2). Based on our observations, we would not recommend $1 \mathrm{~V}$ evaluations as an alternative reading strategy to lower CEM-related radiation exposure.

Since previous studies have demonstrated an improved cancer detection rate in women at high risk for developing breast cancer who were annually screened with breast MRI, many guidelines have recommended its use for this indication. In our national guidelines, annual breast MRI screening is recommended for most women with a known genetic predisposition for developing breast cancer, such as $B R C A-1$, BRCA-2, TP53, PALB2, CDH1, STK11, and PTEN gene mutations. Other women eligible for this kind of screening are those with prior chest (mantle) radiation or with Li Fraumeni or Cowden syndrome. Screening is initiated at the age of 25 or 30 (depending on the gene mutation) and continues to the age of 60 [20]. Consequently, all these women undergo approximately 30-35 screening breast MRI exams in their lives.

Dynamic, gadolinium (Gd)-enhanced T1w images are the backbone of a breast MRI protocol. McDonald et al observed an accumulation of Gd within the brain, even in patients with normal renal function and without any intracranial abnormalities [21, 22]. Although confirmed by other studies, there is no evidence at present to show any adverse effects [8], but several international bodies have recommended a more cautious use of these agents until long-term effects can be ruled out.

CEM might be considered in the future as an alternative screening modality for this group of women, for example for 
Table 2 Overview of histopathological diagnoses of false-negative cases

\begin{tabular}{|c|c|c|c|c|c|c|c|}
\hline Missed on view & Lesion type & Tumor type & Size (mm) & Grade & ER & PR & HER2 \\
\hline $1 \mathrm{~V}$ & Calcifications & DCIS & 8 & 2 & N/A & N/A & N/A \\
\hline $1 \mathrm{~V}$ & Calcifications & DCIS & 12 & 2 & N/A & N/A & N/A \\
\hline $1 \mathrm{~V}$ & Calcifications & DCIS & 10 & 1 & N/A & N/A & N/A \\
\hline $1 \mathrm{~V}$ & Calcifications & DCIS & 25 & 2 & N/A & N/A & N/A \\
\hline $1 \mathrm{~V}$ & Calcifications & DCIS & 14 & 3 & N/A & N/A & N/A \\
\hline $1 \mathrm{~V}$ & Calcifications & DCIS & 30 & 1 & N/A & N/A & N/A \\
\hline $1 \mathrm{~V}$ & Calcifications & DCIS & 5 & 3 & N/A & N/A & N/A \\
\hline $1 \mathrm{~V}$ & Mass & ILC & 13 & 2 & Positive & Positive & Negative \\
\hline $1 \mathrm{~V}$ & Mass & ILC & 6 & 2 & Positive & Positive & Negative \\
\hline $1 \mathrm{~V}$ & Mass & ILC & 5 & 2 & Positive & Positive & Negative \\
\hline $1 \mathrm{~V}$ & Mass & NST & 8 & 2 & Positive & Positive & Negative \\
\hline $1 \mathrm{~V}$ & Mass & Papillary & 4 & 1 & Positive & Positive & Negative \\
\hline $2 \mathrm{~V}$ & Calcifications & DCIS & 20 & 2 & N/A & N/A & N/A \\
\hline Both & Calcifications & DCIS & 10 & 3 & N/A & N/A & N/A \\
\hline Both & Calcifications & DCIS & 15 & 3 & N/A & N/A & N/A \\
\hline Both & Calcifications & DCIS & 48 & 3 & N/A & N/A & N/A \\
\hline Both & Calcifications & DCIS & 8 & 2 & N/A & N/A & N/A \\
\hline Both & Calcifications & DCIS & 12 & 2 & N/A & N/A & N/A \\
\hline Both & Calcifications & DCIS & 2 & 2 & N/A & N/A & N/A \\
\hline Both & Mass & ILC & 15 & 2 & Positive & Positive & Negative \\
\hline Both & Mass & NST & 8 & 1 & Positive & Negative & Negative \\
\hline
\end{tabular}

$E R$, estrogen receptor; $P R$, progesterone receptor; HER2, human epidermal growth factor-2; DCIS, ductal carcinoma in situ; $I L C$, invasive lobular carcinoma; NST, invasive carcinoma of no special type those who have claustrophobia, refuse the repeated administration of Gd-based contrast agents, or who prefer CEM over breast MRI. In CEM, an iodine-based contrast agent is administered intravenously 2 min prior to image acquisition. By using a dual-energy technique, the radiologist can read a low-energy image (which is like a conventional full-field digital mammogram) and a recombined image, in which areas of enhancement can be appreciated (Fig. 3) [23]. Previous studies have shown that CEM is consistently superior to FFDM, with comparable diagnostic performance to breast MRI in terms of both cancer detection and the evaluation of disease extent $[11-13,24]$.

Jochelson et al were the first to evaluate the potential of CEM as a screening tool for high-risk patients in a study containing 307 cases [14]. In the first screening round, three cancers (two invasive and one DCIS) were detected. Breast MRI detected all three, whereas CEM detected only the two invasive breast cancers. None of the cancers were visible on the low-energy images. After the next screening round (after 2 years), five additional screen-detected cancers were observed. The PPV turned out to be comparable between CEM and breast MRI: $15 \%$ and 14\%, respectively. Hence, the authors concluded that CEM might be a suitable alternative for screening these women when they had a contra-indication for breast MRI or who have limited access to it. However, an important limitation of using CEM as a screening tool over MRI is the current lack of (commercially available) CEMguided stereotactic capabilities. This is expected to change soon, as prototypes are currently being evaluated for clinical applications. Nevertheless, they are not available at this point, which further supports the fact that at this point, screening of high-risk women using CEM can only be recommended when breast MRI is contra-indicated.

More recently, Sorin et al studied the diagnostic accuracy of CEM compared with FFDM in women with dense breasts and intermediate breast cancer risk (i.e., positive personal or family history). In this study of 611 cases, the sensitivity increased to $90.5 \%$ when using CEM (for FFDM sensitivity was $52.4 \%$ ), but specificity dropped from $90.5 \%$ (FFDM) to $76.1 \%$ (CEM) [15]. These preliminary studies confirm that CEM has a potential as a screening tool in women with high or intermediate breast cancer risk, or even as supplemental imaging tool in women with dense breasts.

However, an important disadvantage of performing CEM as screening tool is its increased radiation dose. Jeukens et al showed on a single commercially available unit that the radiation dose increased with $81 \%$ when CEM was used instead of full-field digital mammography (mean radiation dose of mammography being $1.55 \mathrm{mGy}$ per exposure, compared with 2.80 mGy per CEM exposure) [16]. Hence, a complete 

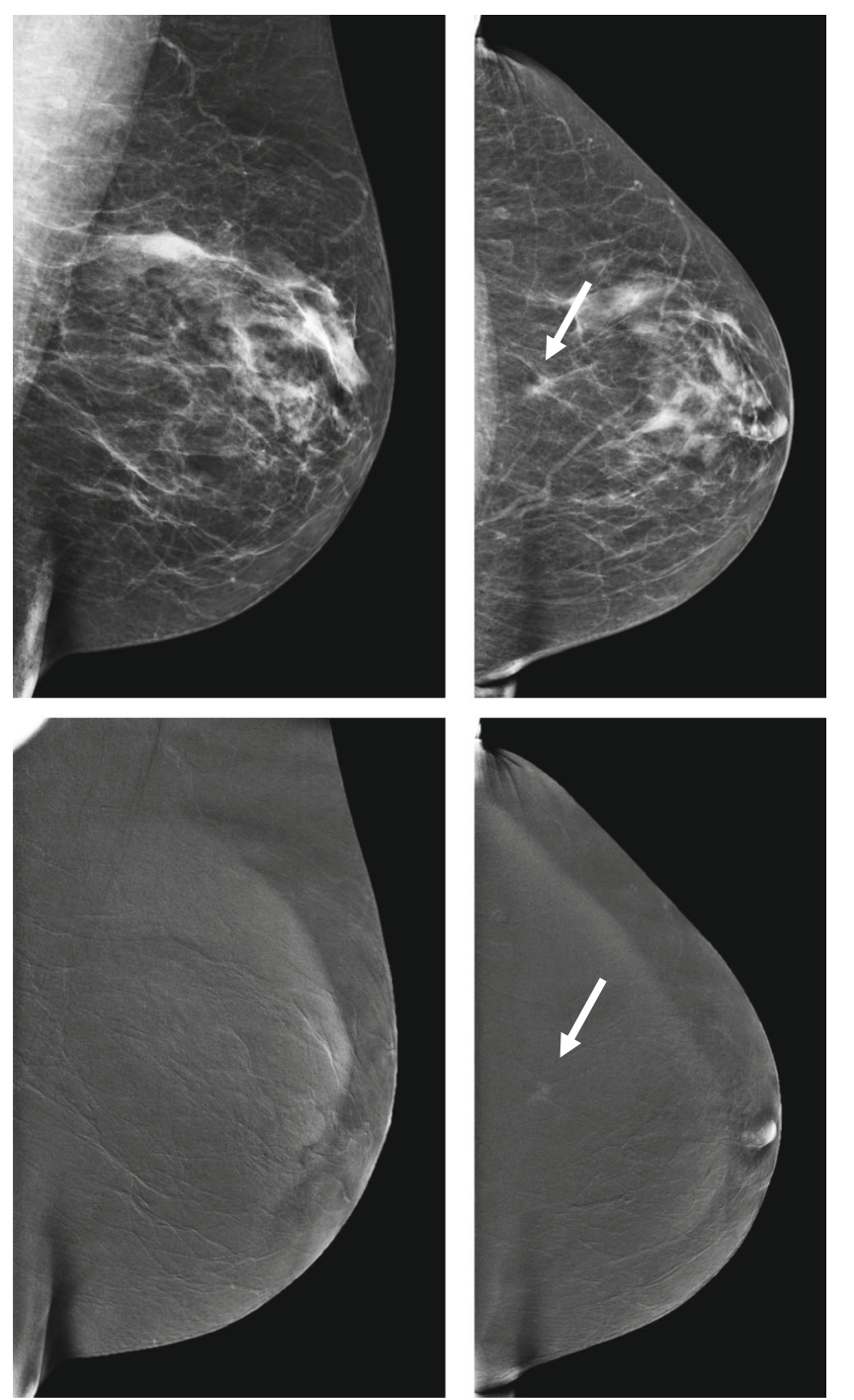

Fig. 1 Example of an invasive lobular cancer in the left breast, not detected in $1 \mathrm{~V}$ (MLO), only detected on $2 \mathrm{~V}$ as an ill-defined focal asymmetry that showed slight enhancement on the recombined images (arrow)

FFDM (i.e., two breasts, two views) would result in an annual dose of $6.2 \mathrm{mGy}$. Considering the lifetime-attributable risk numbers and a 30 -year screening period (according to our current national guidelines), the lifetime risk of radiationinduced breast cancer incidence is estimated to be $0.23 \%$ and its mortality $0.06 \%$ [25]. If CEM would be used as a screening tool, the annual dose would become $11.2 \mathrm{mGy}$, resulting in a lifetime risk of breast cancer incidence and mortality of $0.41 \%$ and $0.1 \%$, respectively, during the 30 -year screening period.

In theory, the use of single-view CEM could provide an interesting alternative, especially if sensitivity would not decrease significantly. The ideal study design to test this hypothesis would be a randomized controlled clinical trial, dividing women in a CEM- and MRI group for screening. However, the breast cancer incidence in this population is very low, requiring a larger number of study participants and a sufficiently long follow-up period to draw any final conclusions. Therefore, we opted to perform a retrospective study designed as a non-inferiority study first to evaluate whether the use of $1 \mathrm{~V}$ did not result in an unacceptable worse sensitivity for detecting breast cancer in our study population, while maintaining similar specificity when compared with $2 \mathrm{~V}$.

With respect to the consensus evaluation, the lower limit of the $90 \% \mathrm{CI}$ around the difference in sensitivity between $1 \mathrm{~V}$ and $2 \mathrm{~V}$ evaluation is $-15 \%$ and lies below the non-inferiority margin of $-10 \%$. However, the entire $90 \% \mathrm{CI}$ ranging from 15 to $-5.3 \%$ does not exclude the non-inferiority margin. Therefore, we cannot conclude that $1 \mathrm{~V}$ is non-inferior to $2 \mathrm{~V}$ with respect to sensitivity, but neither can it be concluded that $1 \mathrm{~V}$ evaluations are inferior to $2 \mathrm{~V}$ evaluations (at a predefined non-inferiority margin of $-10 \%)$. With this chosen non-inferiority margin, formally the results are inconclusive with respect to non-inferiority [26]. However, based on the substantial and statistically significant decrease in sensitivity and overall diagnostic performance, we would not recommend $1 \mathrm{~V}$ CEM as an alternative reading strategy.

The most important causes for FN findings, and thus a decrease in sensitivity, were ILC and DCIS. Due to some selection bias caused by the study design, the prevalence of both ILC and DCIS in malignant cases was higher than would be expected (23.8\% and $33.3 \%$, respectively). Hypothetically, the sensitivity of both strategies would improve if these entities were less frequently observed in this population. Nevertheless, ILC shows no to subtle enhancement on $2 \mathrm{~V}$ CEM exams [27], while Houben et al recently showed that the use of $2 \mathrm{~V}$ CEM does not significantly increase its diagnostic performance for suspicious breast calcifications [28]. In summary, these diagnoses remain challenging even in $2 \mathrm{~V}$ CEM, and it is not plausible that the detection of these lesions will be better on $1 \mathrm{~V}$ CEM.

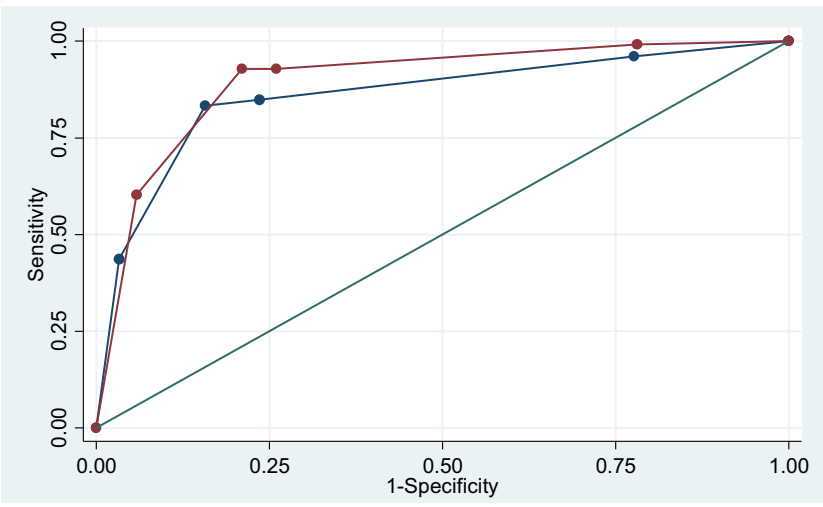

Fig. 2 Receiver operating characteristic (ROC) curves for the consensus results $(\mathrm{R} 1+\mathrm{R} 2+\mathrm{R} 3)$ based on the $1 \mathrm{~V}$ reading strategy (blue line) and the $2 \mathrm{~V}$ reading strategy (red line). The area under the ROC curve (AUC) for $1 \mathrm{~V}$ readings was significantly lower $(0.861)$ than the (standard) $2 \mathrm{~V}$ readings $(0.899$, one-sided $p$ value 0.0174$)$ 
Fig. 3 Typical example of a contrast-enhanced mammography exam, showing the lowenergy images in the top row and the recombined (contrastenhanced) images on the bottom row. In this case, an irregular, illdefined mass is visible in the outer lower quadrant of the right breast (arrow), showing rim enhancement after contrast administration. Biopsy revealed an invasive carcinoma of no special type (NST)
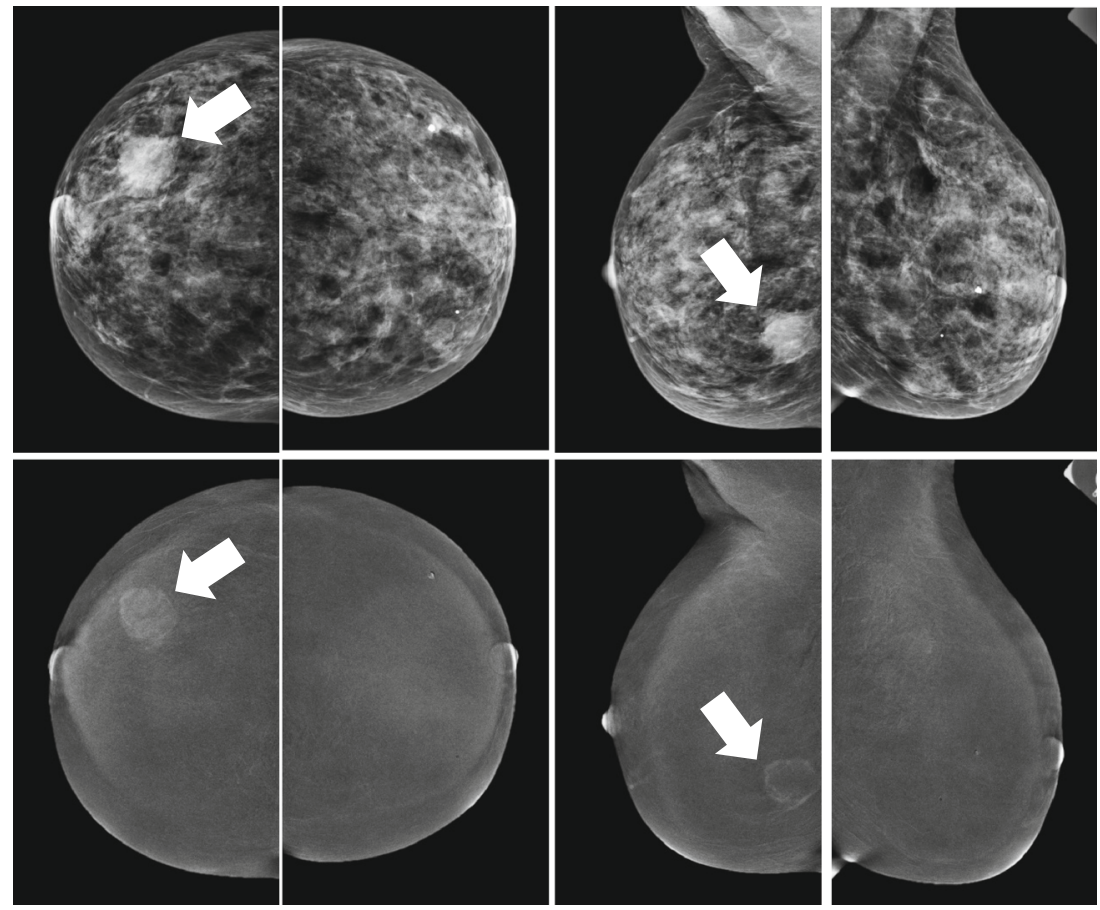

Another important disadvantage of CEM is the use of iodinated contrast agents, which can result in hypersensitivity reactions (or even anaphylactic shock) or can cause contrastinduced nephropathy. In a recent study of 839 patients, the incidence of mild or moderate hypersensitivity reactions during a CEM was $0.6 \%$, without any severe reactions resulting in hospital admission or worse [29]. Although contrastinduced nephropathy might occur as a result of the administration of iodinated contrast agents, the incidence was recently estimated to be $2.6-2.7 \%$ in high-risk patients (i.e., with a glomerular filtration rate of $30-50 \mathrm{ml} / \mathrm{min} / 1.73 \mathrm{~m}^{2}$ ) [30]. The expected incidence in our current population is expected to be lower, as it is not a high-risk group [29].

Considering these facts that CEM requires iodinated contrast agents and must be performed in $2 \mathrm{~V}$, we agree with Jochelson et al that at present, CEM might be considered an alternative to breast MRI for screening high-risk patients, not a replacement. We support their proposal to perform larger prospective trials on this topic, but our results show that the CEM exam used in these studies should consist of a standard, two-view CEM exams of both breasts.

\section{Study limitations}

Our study had several limitations. First, the cohort consisted of women undergoing CEM for an abnormality already suspected using a different modality, introducing some selection bias. However, the readers were blinded for the CEM indication and final diagnosis when reading the exams.
Second, we used a blinded double-reading strategy for the analyses of this study, as it is similar to our national screening program. Other reading strategies, such as unblinded double reading, might have resulted in different observations. Another potential reading strategy, using a single radiologist aided by computer-aided detection (CAD) systems, was not feasible, since there are currently no approved CAD systems available for CEM. Third, the population that we used is not a high-risk population that would be considered for intensified screening (i.e., lifetime risk $>20 \%$ ). These are more often young(er) women, with more often dense breasts, who can additionally express different breast cancer subtypes [31, 32]. CEM might be a suitable alternative screening method, since Lord et al showed a sensitivity of mammography and breast MRI combined of 94\%, with a specificity varying between 77 and 96\% [33]. Although these findings are in line with our observed diagnostic performance of $2 \mathrm{~V}$ CEM exams, it remains unclear how the results of our study population (i.e., a non-screening population not of high risk) would be applicable to the target population of (screening) high-risk patients. The use of CEM for screening high-risk patients needs to be studied further, but then using $2 \mathrm{~V}$ CEM exams (not $1 \mathrm{~V}$ ). Finally, the follow-up period for benign diagnoses should preferably be more than 2 years for all lesions studied, while our current mean follow-up period is 21 months. Nevertheless, in a study using a similar population, Lalji et al showed that the chance of having overlooked a breast cancer when CEM was deemed "negative" is negligible [17]. 


\section{Conclusion}

Non-inferiority of $1 \mathrm{~V}$ evaluations as alternative reading strategy to standard $2 \mathrm{~V}$ evaluations could not be concluded. $1 \mathrm{~V}$ evaluations had lower diagnostic performance compared with $2 \mathrm{~V}$ evaluations.

Funding The authors state that this work has not received any funding.

\section{Compliance with ethical standards}

Guarantor The scientific guarantor of this publication is M.B.I. Lobbes.

Conflict of interest The authors of this manuscript declare no relationships with any companies, whose products or services may be related to the subject matter of the article.

Statistics and biometry One of the authors has significant statistical expertise.

Informed consent Written informed consent was waived by the Institutional Review Board.

Ethical approval Institutional Review Board approval was not required because of the retrospective study design that was used in this article.

\section{Methodology}

- Retrospective non-inferiority study performed at one institution

Open Access This article is distributed under the terms of the Creative Commons Attribution 4.0 International License (http:// creativecommons.org/licenses/by/4.0/), which permits unrestricted use, distribution, and reproduction in any medium, provided you give appropriate credit to the original author(s) and the source, provide a link to the Creative Commons license, and indicate if changes were made.

\section{References}

1. Phi XA, Saadatmand S, De Bock GH et al (2016) Contribution of mammography to MRI screening in BRCA mutation carriers by BRCA status and age: individual patient data meta-analysis. Br J Cancer 6:631-637

2. Saadatmand S, Obdeijn IM, Rutgers EJ et al (2015) Survival benefit in women with BRCA1 mutation or familial risk in the MRI screening study (MRISC). Int J Cancer 137:1729-1738

3. Phi XA, Houssami N, Obdeijn IM et al (2015) Magnetic resonance imaging improves breast screening sensitivity in BRCA mutation carriers age $\geq 50$ years: evidence form an individual patient data meta-analysis. J Clin Oncol 33:349-356

4. Saslow D, Boetes C, Burke W et al (2007) American Cancer Society guidelines for breast screening with MRI as an adjunct to mammography. CA Cancer J Clin 57:75-89

5. Sardanelli F, Boetes C, Borisch B et al (2010) Magnetic resonance imaging of the breast: recommendations from the EUSOMA working group. Eur J Cancer 46:1296-1316

6. Mann RM, Balleyguier C, Baltzer PA et al (2015) Breast MRI: EUSOBI recommendations for women's information. Eur Radiol $25: 3669-3678$
7. Zhang Y, Ren H (2017) Meta-analysis of diagnostic accuracy of magnetic resonance imaging and mammography for breast cancer. J Cancer Res Ther 13:862-868

8. Gulani V, Calamante F, Shellock FG et al (2017) Gadolinium deposition in the brain: summary of evidence and recommendations. Lancet Neurol 16:564-570

9. Weis SM, Cheresh DA (2011) Tumor angiogenesis: molecular pathways and therapeutic targets. Nat Med 17:1359-1370

10. Lobbes MB, Smidt ML, Houwers J, Tjan-Heijnen VC, Wildberger JE (2013) Contrast enhanced mammography: techniques, current results, and potential indications. Clin Radiol 68:935-944

11. Jochelson MS, Dershaw DD, Sung JS et al (2013) Bilateral contrast-enhanced dual-energy mammography: feasibility and comparison with conventional digital mammography and MR imaging in women with known breast carcinoma. Radiology 266: $743-751$

12. Fallenberg EM, Schmitzberger FF, Amer H et al (2017) Contrastenhanced spectral mammography vs. mammography and MRI: clinical performance in a multi-reader evaluation. Eur Radiol 27: 2752-2764

13. Li L, Roth R, Germaine P et al (2017) Contrast-enhanced spectral mammography (CESM) versus breast magnetic resonance imaging (MRI): a retrospective comparison in 66 breast lesions. Diagn Interv Imaging 98:113-123

14. Jochelson MS, Pinker K, Dershaw D et al (2017) Comparison of screening CEDM and MRI for women at increased risk for breast cancer: a pilot study. Eur J Radiol 97:37-43

15. Sorin V, Yagil Y, Yosepovich A et al (2018) Contrast-enhanced spectral mammography in women with intermediate breast cancer risk and dense breasts. AJR Am J Roentgenol 211:W1-W8

16. Jeukens CR, Lalji UC, Meijer E et al (2014) Radiation exposure of contrast-enhanced spectral mammography compared with full-field digital mammography. Invest Radiol 49:659-665

17. Lalji UC, Houben IP, Prevos R et al (2016) Contrast-enhanced spectral mammography in recalls from the Dutch breast cancer screening program: validation of results in a larger multireader, multicase study. Eur Radiol 26:4371-4379

18. Tango $\mathrm{T}$ (1998) Equivalence test and confidence interval for the difference in proportions for the paired-sample design. Stat Med 17:891-908

19. DeLong ER, DeLong DM, Clarke-Pearson DL (1988) Comparing the areas under two or more correlated receiver operating characteristic curves: a nonparametric approach. Biometrics 44:837-845

20. Integraal Kankercentrum Nederland, richtlijn Mammacarcinoom. Available via http://www.oncoline.nl/borstkanker. Accessed 24 Sept 2018

21. McDonald RJ, McDonald JS, Kallmes DF et al (2015) Intracranial gadolinium deposition after contrast-enhanced MR imaging. Radiology 275:772-782

22. McDonald RJ, McDonald JS, Kallmes DF et al (2017) Gadolinium deposition in human brain tissues after contrast-enhanced MR imaging in adult patients without intracranial abnormalities. Radiology 285:546-554

23. Patel BK, Lobbes MBI, Lewin J (2018) Contrast enhanced spectral mammography: a review. Semin Ultrasound CT MR 39:70-79

24. Lobbes MB, Lalji UC, Nelemans PJ et al (2015) The quality of tumor size assessment by contrast-enhanced spectral mammography and the benefit of additional breast MRI. J Cancer 6:144-150

25. National Research Council (2006) Health risks for exposure to low levels of ionizing radiation: BEIR VII phase, 2nd edn. National Academic Press, Washington, DC

26. Piaggio G, Elbourne DR, Pocock SJ, Evans SJ, Altman DG (2012) Reporting of non-inferiority and equivalence randomized trials: extension of the CONSORT 2010 statement. JAMA 308:2594-2604 
27. Van Nijnatten TJA, Jochelson MS, Pinker K, et al (2019) Differences in degree of enhancement on CEM between ILC and IDC. BJR Open 5:20180046

28. Houben IP, Vanwetswinkel S, Kalia V et al (2019) Contrastenhanced spectral mammography in the evaluation of breast suspicious calcifications: diagnostic accuracy and impact on surgical management. Acta Radiol. https://doi.org/10.1177/ 0284185118822639

29. Houben IPL, Van de Voorde P, Jeukens CRLPN et al (2017) Contrast-enhanced spectral mammography as work-up tool in patients recalled from breast cancer screening has low risks and might hold clinical benefits. Eur J Radiol 94:31-37

30. Nijssen EC, Rennenberg RJ, Nelemans PJ et al (2017) Prophylactic hydration to protect renal function from intravascular iodinated contrast material in patients at high risk of contrast-induced nephropathy (AMACING): a prospective, randomized, phase 3, controlled, open-label, non-inferiority trial. Lancet 389:1312-1322
31. Checka CM, Chun JE, Schnabel FR, Lee J, Toth H (2012) The relationship of mammographic density and age: implications for breast cancer screening. AJR Am J Roentgenol 198:W292-W295

32. Ha SM, Chae EY, Cha JH, Kim HH, Shin HJ, Choi WJ (2017) Association of BRCA mutation types, imaging features, and pathologic findings in patients with breast cancer with BRCA1 and BRCA2 mutations. AJR Am J Roentgenol 209:920-928

33. Lord SJ, Lei W, Craft P et al (2007) A systematic review of the effectiveness of magnetic resonance imaging (MRI) as an addition to mammography and ultrasound in screening young women at high risk of breast cancer. Eur J Cancer 43:1905-1917

Publisher's note Springer Nature remains neutral with regard to jurisdictional claims in published maps and institutional affiliations. 